\title{
A FINITE ELEMENT MODEL STUDY ON THE ROLE OF TRUNK MUSCLES IN GENERATING INTRA-ABDOMINAL PRESSURE
}

\author{
NAVid ARJMAnd, AboulfaZl ShiraZi-AdL*, Mohamad PaRnianPour** \\ Department of Mechanical Engineering, Sharif University of Technology, Tehran, Iran \\ ^Department of Mechanical Engineering, Ecole Polytechnique, Montreal, Canada \\ * Department of Industrial, Welding and Systems Engineering, \\ The Ohio State University, Ohio, USA
}

\section{INTRODUCTION}

The significant economic costs and human suffering due to work related low-back disorders have motivated many scientists from diverse disciplines to search for mechanisms of injury and the associated risk factors. Prevention strategies are predicated on the premise that risk factors can be identified, controlled or eliminated by appropriately designed interventions.

The nature of spinal loading (in terms of its magnitude, direction, repetition and duration) has been associated with the development and aggravation of work-related low back disorders (19-22,26-28,33, $34,36,38)$. Hence, the spine has been the focus of many biomechanical evaluations on how external loads are carried and shared by the trunk muscles and osteoligamentous spine. Historically, the limitations in modeling of spinal musculature have led the investigators to search for auxiliary mechanisms assisting the extensor muscles in satisfying the equilibrium conditions $(1,3,8,13,18,26-28,30)$. The detailed description of these theories and their biomechanical evaluations (testing the validity of claims regarding the unloading function of the intra-abdominal pressure and utilization of lumbodorsal fascia) can be found in a recent review by McGill and Norman (28). It is now recognized that the stability and equilibrium conditions of spine must be simultaneously insured by proper neuromuscular functions of various components of spine $(1,4,7,8,12,15,17,330,33-36,40)$.

Received: March 13, 2001; accepted: June 15, 2001

Correspondence: A. Shirazi-Adl, Ph.D.

Dept of Mechanical Engineering, Ecole Polytechnique PO Box 6079, Station centre-ville, Montreal, Quebec, Canada H3C 3A7

E-mail: abshir@meca.polymtl.ca
While we have advanced in anatomical modeling of posterior spinal musculature due to reported pioneering investigations (2), we still lack a similar precision about the anterior trunk muscles despite some recent improvements $(29,32,39)$. The transverse abdominis, internal and external oblique muscles are flat muscles. The line of action of oblique muscles also varies around the trunk. Hence, representing oblique muscles with a single equivalent muscle is likely inadequate from both mechanical and neural control perspectives (11). There is evidence that different portions of oblique muscles may be selectively activated (11). The role of transverse abdominis has been neglected in most equilibrium-based models since its horizontal orientation covering the anterior abdominal wall limits its direct moment generation capability about the lumbar spine. Its location beneath internal and external obliques has also made it inaccessible for the noninvasive surface electrodes favored in biomechanical studies.

Many have evoked the role of intra-abdominal pressure (IAP) in equilibrium considerations by unloading of spine and assisting the extensor muscles during lifting tasks (8). More recently, the concept of higher IAP contributing to the stability of spine by stiffening of spinal column has been proposed $(1,2,40)$.

Clinical studies have shown that there are significantly higher delays in recruitment of transverse abdominis (TA) muscles during the self-generated postural perturbations that is caused during the movement of lower limbs $(15,16,34)$ in low back pain patients. The activities of TA, however, precede the activities of other trunk muscles and the primary movers of the lower limbs in normal subjects. Based on assumed importance of neural control of TA, a special theory and treatment strategy for segmental stabilization of spine by trunk muscles has been developed with significant positive results in practice (34). A quantitative assessment of the role of TA in the spine mechanics is 
never-the-less missing.

The aforementioned void motivated the development of the following model as a first step towards a comprehensive validated model of the human trunk. The muscles are modeled as continuous fiberreinforced membranes that surround the intraabdominal cavity. These membranes, unable to carry any moments, can resist tension primarily along their fiber direction; they develop in-plane stresses while generating IAP. The finite element model is used to allow the varying distribution, cross-sectional area and fiber direction of muscles around the trunk. Hence, this model improves upon the Daggfeldt and Thorstensson model (8) that considered only one fiber orientation and muscle type covering the whole trunk. While the model is still idealized due to its lack of passive spine and anatomical details, it is a first step towards a novel approach to modeling of abdominal muscles. It was thought that evolution of the model should take a systematic step to allow us to appreciate the contribution of each added element. The ultimate goal of this modeling effort is to allow realistic appraisal of the role of trunk muscles in maintaining the equilibrium and stability of spine under normal daily and occupational activities as well as clinical conditions.

\section{METHOD}

\subsection{Anatomical Description of Trunk Muscles}

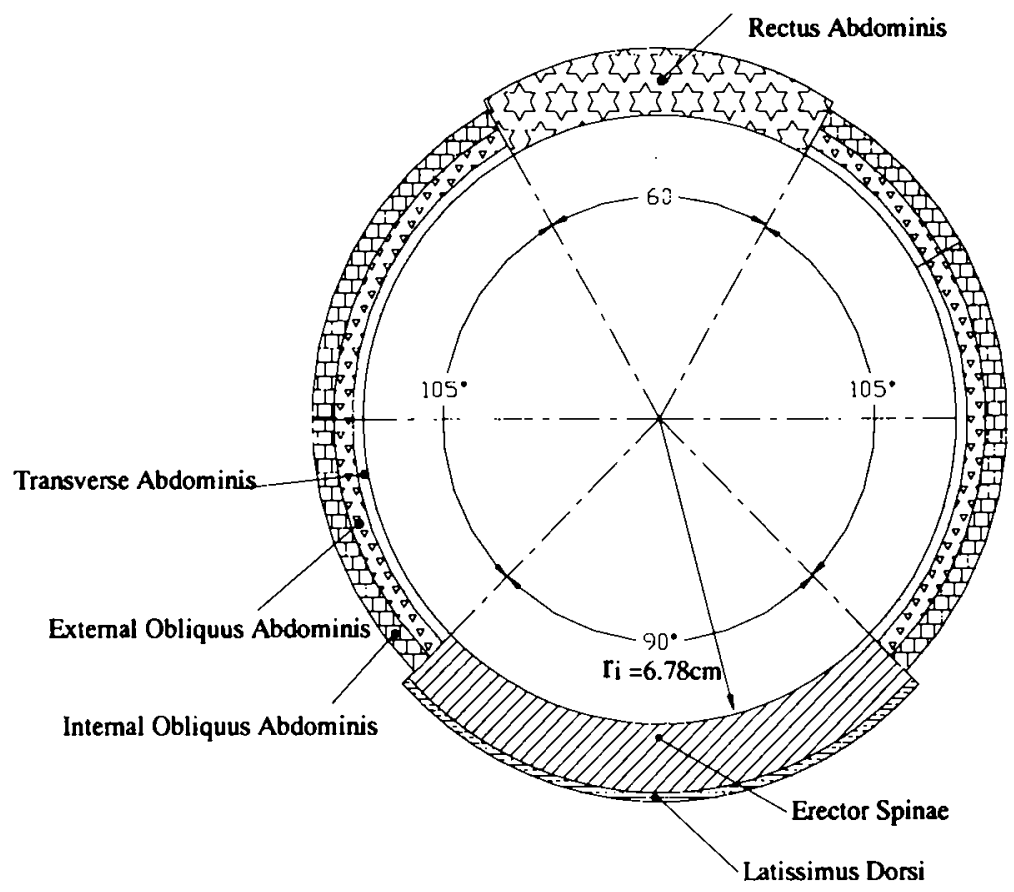

Fig. 1- Arrangement of muscles around the intra-abdominal cavity. The thickness and distribution of each muscle is based on the literature cited in Table 1.
The abdominal wall was assumed to be a cylindrical membrane (8) with a radius of $6.78 \mathrm{~cm}$ based on the average peritoneal cavity of $144.4 \mathrm{~cm}^{2}$ at the L4/L5 (27). The height of cylinder has been considered as the breath of transverse abdominis that is about $11 \mathrm{~cm}$ based on a cadaver study (27). The six muscle groups surrounding the abdominal wall are the erector spinae (ES), latissimus dorsi (LAT), rectus abdominis (RA), internal obliquus (IOB), external obliquus (EOB), and transverse abdominis (TA) (Fig. 1). The appropriate thickness was computed for each muscle based on their anatomical cross sectional areas (Table 1) and their circumferential distribution (Fig. 1). Fibers orientation for RA and ES has been taken in the longitudinal direction and in the transverse direction for TA whereas that for IOB, EOB and LAT has been selected from the study of Nussbaum et. al. (32) as shown in Fig. (2). Hence, each muscle has its own specific thickness, circumferential distribution and fibers orientation.

\subsection{The Finite Element Model}

A finite element (FE) model has been developed for assessing the effect of activation in each muscle individually or in combination on the IAP (Figures 3 and 4). The model was developed and analyzed using ANSYS 5.4 (ANSYS Inc., Southpointe, 275 Technology Drive, Canonsburg, PA, USA). The intra-abdominal cavity was assumed to contain a nearly incompressible fluid (with the bulk modulus of $250 \mathrm{MPa}$ ) surrounded 
Table 1. Anatomical cross sectional area (ACSA) and computed thickness of trunk muscles

\begin{tabular}{|l|c|c|c|}
\hline Muscle & $\begin{array}{c}\text { ACSA } \\
\left(\mathrm{cm}^{2}\right)\end{array}$ & $\begin{array}{c}\text { Computed } \\
\text { Thickness } \\
(\mathrm{mm})\end{array}$ & Reference \# \\
\hline Rectus Abdominis (RA) & 20 & 23.94 & 5 \\
\hline Latissmus Dorsi (LAT) & $2 \times 4$ & 2.474 & 6 \\
\hline Internal Obliquus (IOB) & $2 \times 9$ & 6.496 & 6 \\
\hline External Obliquus (EOB) & $2 \times 10$ & 6.641 & 6 \\
\hline Erector Spinae (ES) & 45.1 & 33.882 & 27 \\
\hline Transverse Abdominis (TA) & 5 & 4.545 & 29 \\
\hline
\end{tabular}

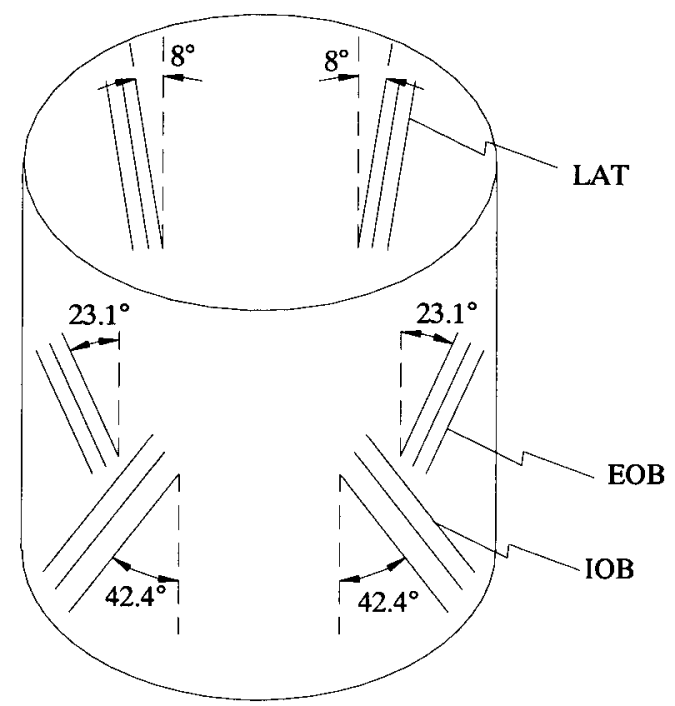

Fig. 2 Angle of fibers of IOB, EOB and LAT taken from Nussbaum et al. (1995). The ES and RA are aligned vertically while $T R$ is aligned horizontally.

by three layers of membrane (Fig. 3). Each layer represented a muscle with transverse isotropic material properties with greater stiffness (Young's modulus) in fibers direction (37). Thickness of each layer and material principal directions ( $\mathrm{x}$-axis being in fibers direction) have been selected as given in Table 1 . For example TA is a membrane layer with thickness of 4.545 $\mathrm{mm}$ and greatest stiffness in transverse direction and RA is modeled as 3 layers with total thickness of 23.94 $\mathrm{mm}$ with primary stiffness in longitudinal direction. The three flat muscles (IOB, EOB, TA) have been represented with three overlapping layers with specific fibers direction and thickness. Also ES is modeled as two layers with total thickness of $33.882 \mathrm{~mm}$ and LAT as one layer covering the ES (Fig. 4).

The top plate simulates the active and/or passive roles of diaphragm through its vertical displacement. When the plate moves downward, the cavity-filled fluid offers resistance and IAP increases generating forces in surrounding muscles. The membrane element is a spatial 4-node plane stress element having in-plane stiffness but no bending (out-of-plane) stiffness. The element has three degrees of freedom at each node (translations in the $\mathrm{x}, \mathrm{y}$ and $\mathrm{z}$ directions). The cavityfilled fluid element is modeled by eight-node elements (3D contained fluid elements with no net flow) having three degrees-of-freedom at each node (similar to membrane elements). Brick elements have been used to model the solid structure of the top plate. Fluid nodes at the boundary coincide with the membrane nodes and are coupled to them only in the radial direction normal to the interface. The nodes at the bottom surface of the top plate are coupled to fluid nodes in the longitudinal direction. Hence, when the plate moves downward and the IAP increases, an upward force is generated acting on the diaphragm. All three layers of membrane elements are connected to each other at nodes by radial-oriented links allowing uncon- 


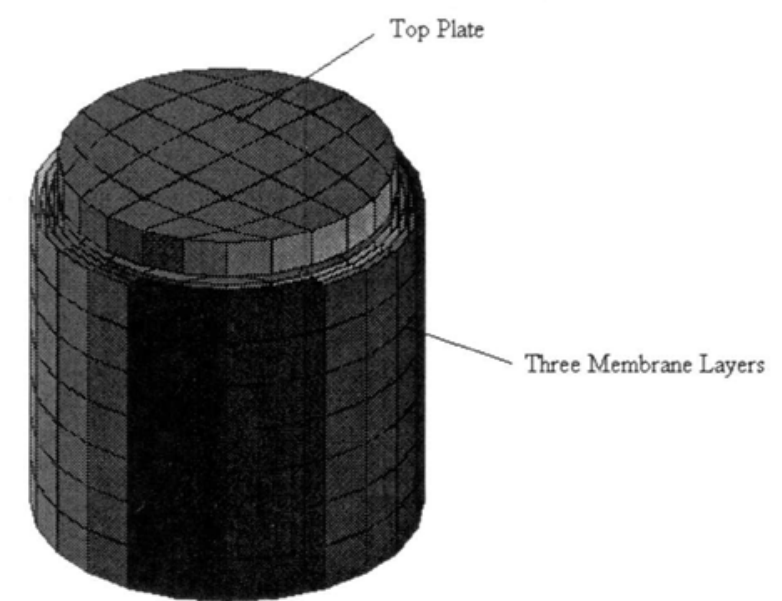

Fig. 3a

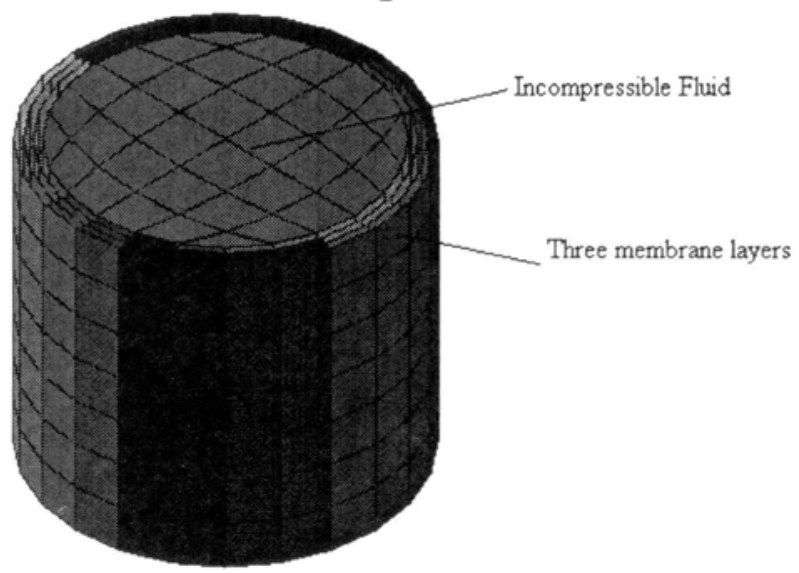

Fig. 3b

Fig. 3 The FE model of intra-abdominal cavity. The model consists of nearly incompressible fluid contained by three layers of membrane simulating the muscles of the abdominal wall. The top plate displaces vertically to simulate the role of diaphragm.

strained relative motions along circumferential and axial directions. The top and bottom membrane nodes are fully constrained. The top plate is displaced downward at a prescribed magnitude causing membrane force/stress in the muscles, hydrostatic pressure (IAP), and constraint (reactive) force on the top plate.

\subsection{Material Properties}

Fiber layers of muscles are considered as membranes with direction-dependent material properties. The transverse isotropy is assumed in the plane normal to the direction of fibers. Each membrane has five independent moduli: the Young's modulus of membrane in the fibers direction $\left(E_{x}\right)$, in the transverse directions $\left(E_{y}=E_{z}\right)$, the shear modulus $\left(G_{x y}=G_{x z}\right)$, and two

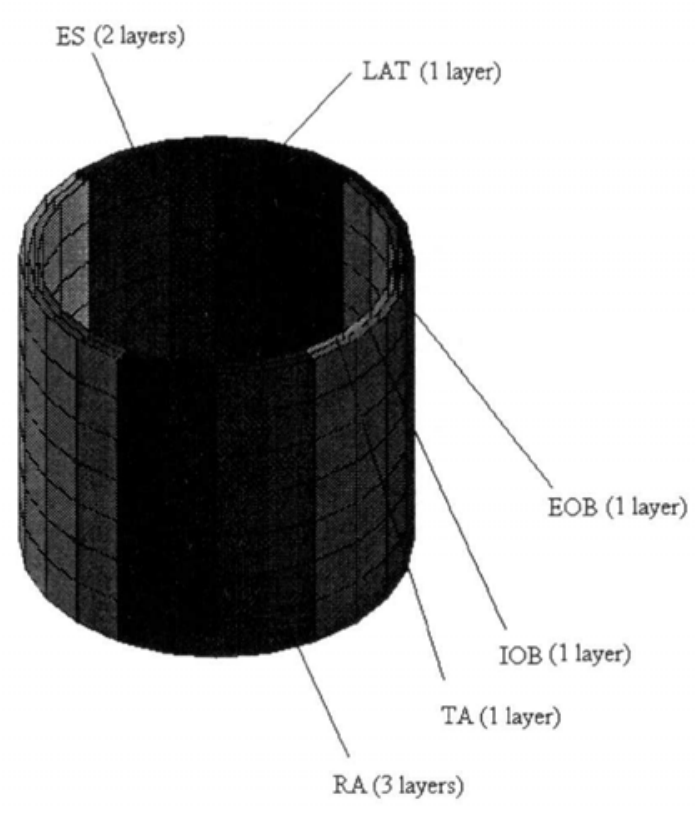

Fig. 4 Three membrane layers representing the trunk muscles.

Poisson's ratios $\left(v_{x y}=v_{x}\right.$ and $\left.v_{p}\right)$. For the inactivated muscle, the following material properties is considered (12):

$$
\begin{aligned}
& E_{x}=0.4 M P a \\
& E_{y}=E_{z}=E_{y} / 3 \\
& G_{x y}=G_{x z}=E_{y} / 2 \\
& V_{x y}=V_{x z}=0.45 \\
& V_{y z}=0.75
\end{aligned}
$$

\subsection{Simulations: Quiet Standing and Valsalva Maneuver}

The IAP in the quiet standing position has been reported to be $0.98 \mathrm{KPa}(31)$. When all muscles are inactivated, the top plate is moved downward until the IAP reaches $0.98 \mathrm{Kpa}$ predicted at $3.15 \mathrm{~mm}$ displacement. This position of the top plate was kept constant for all our simulations in this investigation. Maximum IAP has been considered $3.95 \mathrm{KPa}$ for partial valsalva maneuver (12). Considering the muscles as variable stiffness elements, the Young's modulus along all muscle fibers was subsequently increased until the IAP reached $3.95 \mathrm{KPa}$. This IAP was generated at $E_{x}=1.612 \mathrm{MPa}$ while remaining moduli were scaled accordingly with proportions stated in the previous section. This computed value is referred to as $E_{v}$. 


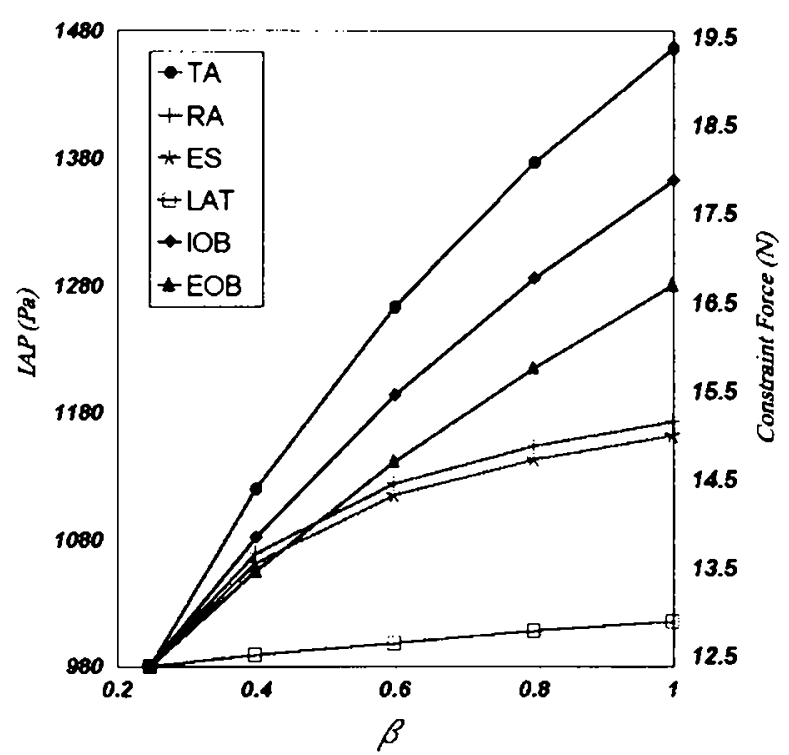

Fig. 5 Predicted variation of IAP (Pa) and the upward (constraint) force (N) that acts on the top plate versus $\beta$; the relative activation of individual muscles

\subsection{The Effect of Variation in Individual Muscle Activation on the IAP}

For determining the effect of each muscle on the IAP, a parameter $\beta$ was defined as:

$$
\beta=\frac{E}{E_{V}} \quad \text { for } \quad \beta_{\text {Min }}<\beta<1
$$

where $E$ is the Youngs's modulus in fibers direction of each muscle and $E_{v}$ is the upper-limit value that was obtained simulating the valsalva maneuver (1.612 $\mathrm{MPa}) ; \beta_{\text {Min }}=0.248$ calculated as the ratio of modulus for the inactivated muscle (lower-limit value of 0.4 $\mathrm{MPa})$ and the $E_{v}$. Hence, $\beta$ is changed from $\beta_{\text {uin }}$ to 1 for different muscles, individually or in combination, and the analysis is performed for each value of $\beta$. The value of $\beta$ indicates the activity level of muscles relative to that during valsalva maneuver (i.e., the upper-limit). It must be noted that none of the muscles were found to reach their full activation capacity during valsalva maneuver (31). Additional cases were also performed to study the effect of changes in muscle fibers direction on the IAP for the three flat muscles at $\beta=1$. The muscle membranes were all verified to remain in tension in all directions.

\section{RESULTS}

The IAP increases with increase in activation level, $\beta$, for each muscle (Fig. 5). The muscles in the

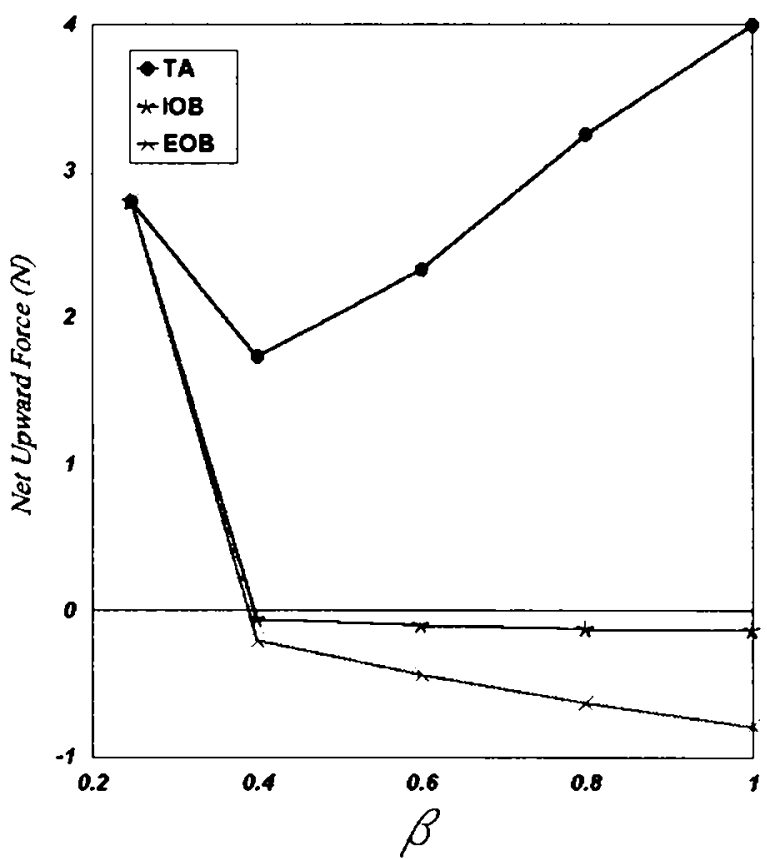

Fig. 6 The unloading effect of IAP due to various activation levels of TA, IOB and EOB.

descending order of their efficiency to generate IAP at $\beta=1$ are predicted to be TA, IOB, EOB, RA, ES, and LAT. The upward constraint force at the top plate shows similar trends for different muscle activation levels exhibiting a nonlinear response for muscles with longitudinal fibers direction (RA and ES) (Fig. 5). The direct unloading force due to higher IAP can be quantified as the difference between the foregoing constraint force and the longitudinal component of the muscle fibers force. It is shown that only TA can create an unloading force due to its horizontally directed muscle fibers (Fig. 6). The rest of muscles generates longitudinal forces greater than the unloading force they create by their contribution through the LAP. The maximum fibers stress (at $\beta=1$ ) remains well below the maximum permissible stresses in all muscles (Fig. 7).

The complexity of the effects of muscle functional anatomy and architecture on the IAP generation capability is shown in Fig. 8. While the EOB and IOB, if with fibers aligned horizontally, would generate more IAP than TA (due to their larger cross sectional area), they are less efficient in their more realistic fiber angles.

The effect of different synergistic recruitment of muscles in generating IAP is depicted in Fig. 9. It is clear that a desired level of IAP can be achieved by different recruitment patterns. However, the compressive penalty of these different recruitments will significantly vary. It is observed that the TA (Fig. 6) is the only single muscle that contributes to a net upward 


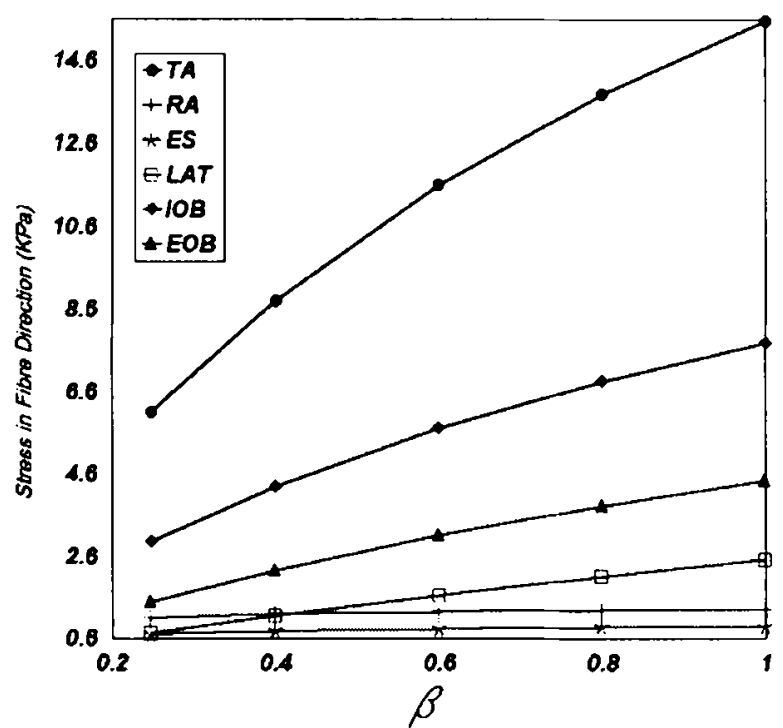

Fig. 7 Tensile stress in fiber direction for different levels of activation in individual muscles.

longitudinal force while the other muscles individually or with synergistic recruitment would likely add to net compressive load on the spine. The other observation that is worth considering with caution is the inability of some combination of muscles (e.g. LAT and ES) to raise sufficient IAP to match the IAP generated by the TA alone. The combined activation of IOB and EOB at valsalva activation level only slightly surpassed the IAP produced by TA alone (Figs. 5 and 9). The clinical implications of these observations must await till a more realistic representation of the passive spine is included and the biomechanical fidelity of the model is enhanced.

\section{DISCUSSION}

The idea that pressure within the trunk might assist unloading the spine was suggested in the 1920 s (18). The underlying theory is that when, for example, a weight is lifted and a flexion moment develops about the torso, this moment is primarily counterbalanced by the posterior back muscles. The pressure in the trunk cavities assists in this respect, producing an extension moment. Two factors responsible for over estimation of IAP role has been the large cross sectional area of diaphragm and the large moment arm of the net upward force acting on the diaphragm (28). The FE approach in this study obviates the need to assign a priori a moment arm to the net upward force which are computed as the displacement at the top plate is prescribed.

Due to the fact that higher IAP is present when the spine is subject to different external moments, the role of IAP must be more complex than the reduction

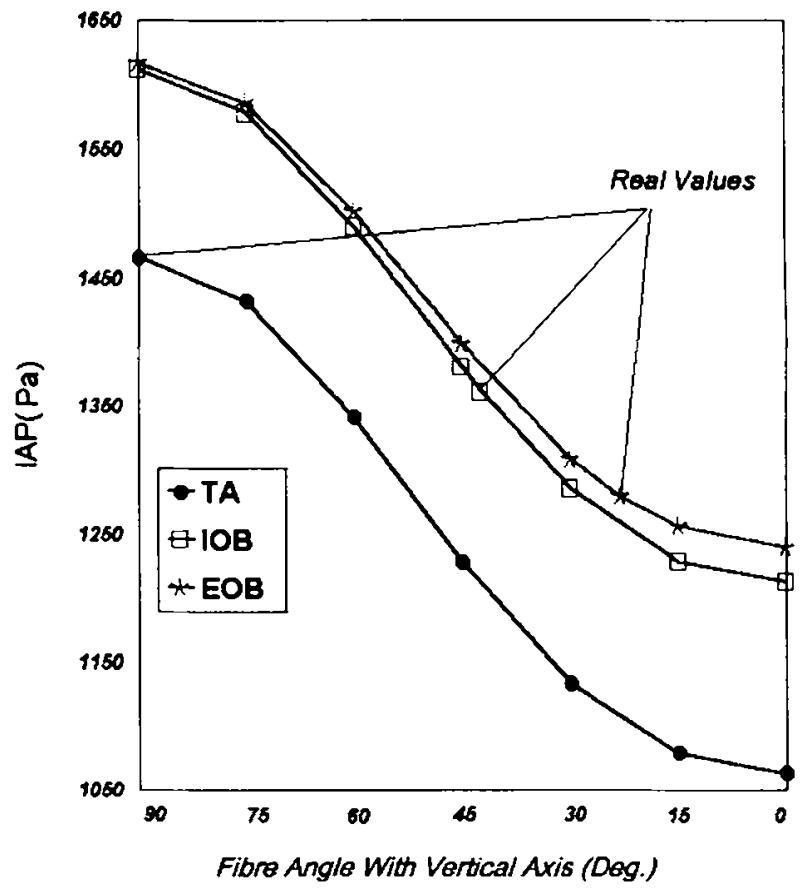

Fig. 8 Variation in IAP (Pa) as a function of fiber angle from transverse $\left(90^{\circ}\right)$ to longitudinal $\left(0^{\circ}\right)$ orientations.

of the extensor moment on the spine (14). Indeed, TA activity is observed with different levels and directions of external moments (34). An increase in the IAP is commonly observed when a large load is placed on the spine during daily activities like lifting, jumping and running $(3,9,10,23,26)$. The function of this increase in pressure is still not well understood. McGill and Norman (28) stated that the possible unloading of the spine by an increased IAP would be counteracted by an equally large or even larger loading by the activated abdominal musculature. On the other hand, some other investigators $(3,8,40)$ claim that IAP can reduce the compression load on the spine. Currently, IAP is generally thought to provide stability to the lumbar spine $(4,7,25)$. The co-contraction together with elevated IAP can effectively stiffen the spine to achieve required stability level $(4,26)$.

The purpose of the present study was to establish a FE model to illustrate the effect of trunk muscles, individually or in combination, on the LAP. The force acted on the diaphragm and resulting stresses and forces in the muscles have also been calculated. In previous studies $(22,32,38)$, each muscle was considered as a single force vector. Obviating this deficiency, we considered each muscle as a membrane layer with greater stiffness in the fibers direction. To assess which muscle can unload the lumbar spine, we followed the method of Daggfeldt and Thorstensson (8). They suggested that for unloading the lumbar spine the 


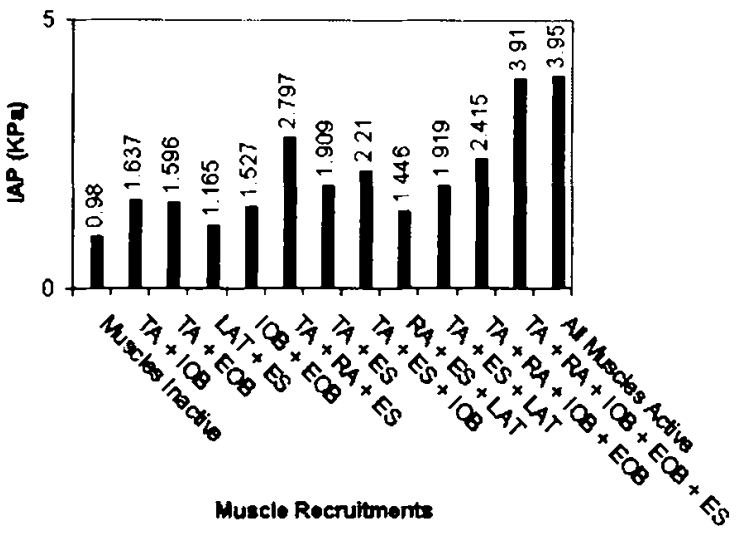

Fig. 9 The effect of different combinations of muscle recruitment patterns at $\beta=1$ on the generated LAP (KPa).

beneficial effects of increasing the IAP (computed constraint force, $F_{C}$ ) need to be greater than the negative effects due to the increased longitudinal tension in the intra-abdominal wall. The difference between $F_{C}$ and $\mathrm{F}_{\mathrm{L}}$ (increase in longitudinal tension in wall due to activity of the same muscle) is shown in Fig. 6. Longitudinal tension has been calculated from reaction forces on the fixed nodes at the top end of membrane layers. Results show that EOB cannot unload the lumbar spine. Daggfeldt and Thorstensson (8) in their simple model of intra abdominal cavity stated that muscles with fibers direction greater than approximately $55^{\circ}$ with the vertical will contribute to unloading while tension in muscles with fibers direction smaller than $55^{\circ}$ will load the lumbar spine. In our model IOB has $42.4^{\circ}$ fibers direction and is more efficient in generating IAP than EOB with smaller angle. It may unload the spine at very low activation levels and achieves almost very little compressive penalty at higher activation levels. However, TA remains the only muscle to unload the lumbar spine at all activation levels considered.

The relatively simple model presented here has a number of limitations which will be delineated after a brief review of functional anatomy of intra-abdominal cavity based on Tortora (41). This section is included to highlight what significant structures are lacking in the current model and to point towards future developments. The posterior abdominal wall consists of the lumbar vertebrae, parts of ilia, psoas major, iliacus and quatratus lumborum muscles. The anterolateral abdominal wall is composed of fasica and four pairs of muscles: EOB, IOB, TA and RA. The first three muscles are flat muscles whereas the last one is a straplike vertical muscle. The fibers of EOB run medially and inferiorly and those of IOB run laterally and inferiorly (almost at a right angle with EOB) while most of the fibers of the TA muscle are horizontally oriented around the abdomen. The RA extends from the pubic crest and pubic symphysis to the cartilage of fifth to seventh ribs and xiphoid process. The aponeuroses of the EOB, IOB and TA muscles form the rectus sheath that encloses the RA muscles to join a tough, fibrous band called linea alba (white line) that extends the xiphoid process of sternum to the pubic symphysis. The moment arms of these muscles have been augmented (29) since they are greatest at the point of junction to form the linea alba.

These anterolateral abdominal muscles have the following functions: to contain and protect abdominal viscera; to sagittally flex, laterally bend and axially rotate the spine; to compress the abdomen during forced expiration, and providing the force for defecation, urination and childbirth. The anterolateral abdominal wall can contract and distend much more than the relatively bulkier and more stable posterior abdominal wall. Ventilation (inspiration and expiration) occurs by changing the size of the thoracic cavity. The most important muscle in ventilation is the diaphragm which with its dome-shaped musculotendinous shape separates the thoracic and abdominal cavities. The two parts of diaphragm are the centeral and peripheral portions. During the inhilation (inspiration) the central (tendinious) dome region moves down while the peripheral (muscular portion) is attached to the lumbar vertebrae, sternum, and costal cartilages. The additional functions of downward movement of central dome of diaphragm include pressurization of intraabdominal cavity in conjunction with the anterolateral abdominal muscle, and assist in returning to the heart the venous blood passing through the abdomen.

It is clear that the present downward direction of the top plate to simulate the action of diaphragm is a gross simplification. The insertion of diaphragm muscle to the passive spine and the rib cage must be properly considered to create more realistic boundary conditions for future developments. The presence of challenges due to breathing or holding of the breath should also be considered for full appraisal of their biomechanical consequences as studied experimentally (2325).

In short, the model developed has the following limitations and its results must be used with caution:

- Lack of passive osteoligamentous spine to share the external load transmitted to spine (e.g. during lifting); consequently lack of attachment points by which the internal loads are transmitted to the spine. Local muscles specially the multifidus must be included for evaluation of the local and global stability of spine.

- Lack of physiological boundary conditions for the floor and ceiling of abdominal cavity and attachments of diaphragm. 
- Idealization of geometry as a cylinder.

- Lack of consideration of different fiber directions in each of muscle groups. It is known that the fibers of these flat muscles (IOB and EOB) may vary across the abdominal wall.

- Imprecise material properties which could influence the predictions.

Future models would need to include the passive spine and its proper interface with the abdominal and trunk muscles so that the external loads would be balanced by the proper activation of these muscles and full assessment of consequence of alternative recruitment patterns could be considered. The method of activation of the muscles may take more direct approaches such as optimization, EMG-driven modeling or kinematics based FE modeling $(5,6,35,36,38)$. The absolute value of predictions could change when the model is refined but it is likely that the relative efficiency of TA shall remain valid. The serial CT scans or MRI should provide more physiological anatomical geometry to be included in future developments. Authors present this paper only as a first step towards providing justifications and feasibility to undertake more advanced studies. Subtle changes in the motor control of low back pain patients and their effects on IAP and alteration in temporal and amplitude patterns of muscles can only be assessed by complex models that could accurately represent the interactions of the neural control, passive and active spinal subsystems.

\section{ACKNOWLEDGEMENTS}

The work is supported by the IRRST-Québec and NSERC-Canada.

\section{REFERENCES}

1. Aspend RM: The spine as an arch. A new mathematical model. Spine 1998.13: 266-274.

2. Bogduk N, Macintosh JE, Pearcy MJ: A universal model of the lumbar back muscles in the upright position. Spine 1992,17(8):897-913.

3. Cholewicki J, Krishna J, McGill SM: Intraabdominal pressure mechanism for stabilizing the lumbar spine. J. Biomechanics 1999,32(1), 13-17.

4. Cholewicki J, McGill SM, Mechanical stability of the in vivo lumbar spine: Implications for injury and chronic low back pain. Clinical Biomechanics 1996,11:1-15.
5. Cholewicki J, McGill SM: EMG assisted optimization: A hybrid approach for estimating muscle forces in an indeterminate biomechanical model. J. Biomechanics 1994, 27(10), 1287-1289.

6. Cholewicki J, McGill SM, Norman RW: Comparison of muscle forces and joint load from an optimization and EMG assisted lumbar spine model: Towards development of a hybrid approach. J. Biomechanics 1995, 28(3), 321-331.

7. Cresswell AG, Oddsson L, Thorstensson A: The influence of sudden perturbations on trunk muscle activity and intra-abdominal pressure while standing. Experimental Brain Research 1994, 98:336341.

8. Daggfeldt $\mathrm{K}$, Thorstensson A: The role of intraabdominal pressure in spinal unloading. J. Biomechanics 1997,30(11/12), 1149-1155.

9. David GC: Intra-abdominal pressure measurements and load capabilities for females. Ergonomics 1985, 28, 345-358.

10. Davis PR: Posture of the trunk during the lifting of weights. British Medical Journal 1959,1, 87-89.

11. Davis JR, Mirka GA: Transverse-contour modeling of trunk muscle-distributed forces and spinal loads during lifting and twisting. Spine. $2000 ; 25(2): 180$ 9.

12. Dietrich M, Kedzior $K$, Zagrejek T, Modelling of muscle action and stability of the human spine. In: Winter JM, Woo SL eds. Multiple Muscle Systems: Biomechanics and Movement Organization. Springer Verlag, New York, 1990, pp. 451-460.

13. Gracovetsky S: Potential of lumbodorsal fascia forces to generate back extension moments during squat lifts. J Biomed Eng. 1989,11(2):172-5.

14. Grew ND: Intraabdominal pressure response to loads applied to the torso in normal subjects. Spine 1980, 5:149-154.

15. Hodges PW, Richardson CA: 1997 Contraction of the abdominal muscles associated with movement of the lower limb. Phys Ther. 1997, 77(2):132-42.

16. Hodges PW, Richardson CA: Delayed postural contraction of transversus abdominis in low back pain associated with movement of the lower limb. J Spinal Disord. 1998, 11(1):46-56.

17. Hodges PW, Richardson CA: Inefficient muscular stabilization of the lumbar spine associated with low back pain. A motor control evaluation of transversus abdominis. Spine 1996,21(22):2640-50.

18. Keith A: Mans Posture: Its evolution and disorders. Lecture IV. The adaptations of the obdomen and its viscera to the orthograde posture. Biritish Medical Journal 1923, 1, 587-590.

19. Kelsey JL, Githens PB, White AA, et al.: An epidemiologic study of lifting and twisting on the job and risk for acute prolapsed lumbar intervertebral disc. J Orthop Res. 1984, 2(1):61-6.

20.Lotz JC, Chin JR: Intervertebral disc cell death is 
dependent on the magnitude and duration of spinal loading. Spine 2000, 25(12):1477-83.

21. Lotz JC, Colliou OK, Chin JR, Duncan NA, Liebenberg E: Compression-induced degeneration of the intervertebral disc: an in vivo mouse model and finite-element study. Spine 1988, 23(23):2493-506.

22. Marras WS, Granata KP: The development of an EMG-assisted model to assess spine loading during whole-body free-dynamic lifting. J. Electromyogr. Kinesiol, 1997, 7(4), 259-268.

23. McGill SM, Sharratt MT, Seguin JP: Loads on spinal tissues during simultaneous lifting and ventilatory challenge. Ergonomics. 1995,38(9):1772-92.

24. McGill SM, Norman RW, Sharratt MT: The effect of an abdominal belt on trunk muscle activity and intra-abdominal pressure during squat lifts. Ergonomics 1990,33(2): 147-60.

25. McGill, SM, Sharratt MT: Relationship between intra-abdominal pressure and trunk EMG. Clinical Biomechanics 1990,5, 59-67.

26. McGill SM: The biomechanics of low back injury: Implications on current practice in industry and the clinic. J. Biomechanics 1997,30, 465-475.

27. McGill SM, Patt N, Norman, RW: Measurement of the trunk musculature of active male using CT scan radiography: Implications for force and moment generating capacity about the L4/L5 joint. J. Biomechanics 1988, 21(4), 329-341.

28. McGill SM, Norman RW: 1993 Low back biomechanics in industry: the prevention of injury through safer lifting. In Current Issues in Biomechanics Grabiner MD (Ed), Human Kinetics, Champaign: IL. 1993, pp 69-120.

29. McGill SM: A revised anatomical model of the abdominal musculature for torso flexion efforts. J. Biomechanics 1996, 29(7), 973-977.

30. Morris JM, Lucas DB, Bresler B: Role of the trunk in stability of spine. J. Bone Joint Surgery 1961,43
A, 327-351.

31. Nachemson A., Andersson GBJ, Schultz AB: Valsalva maneuver biomechanics effects on lumbar trunk loads of elevated intra-abdominal pressures. Spine 1988,11(5), 476-479.

32. Nussbaum MA, Chaffin DB, Catherine JR: Muscle line-of-action affect predicted forces in optimization-based spine muscle modeling. J. Biomechanics 1995,28(4), 401-409.

33. Panjabi MM: The stabilizing system of the spine. Part 1. Function, dysfunction, adaptation, and enhancement. J Spinal Disord. 1992,5(4):383-9

34. Richardson C, Jull G, Hodge P, Hides J: Theraputic exercise for spinal segmental stabilization in low back pain. London: Churchill Livingstone 1999.

35. Shirazi-Adl A, Pamianpour M: Load-bearing and stress analysis of the human spine under a novel wrapping compression loading Clin Biomech (Bristol, Avon). 2000,15(10):718-25.

36. Shirazi-Adl A, Parnianpour M: Effect of changes in lordosis on mechanics of the lumbar spine-lumbar curvature in lifting. J Spinal Disord. 1999,2(5):43647.

37. Shirazi-Adl A. On the fibre composite material models of disc annulus--comparison of predicted stresses. J Biomech. 1989;22(4):357-65.

38. Sparto PJ, Parnianpour M: An electromyographyassisted model to estimate trunk muscle forces during fatiguing repetitive trunk exertions. J Spinal Disord. 1999,12(6):509-18.

39.Stokes IA, Gardner-Morse M: Quantitative anatomy of the lumbar musculature. $J$ Biomech. 1999,32(3):311-6.

40. Thomson KD: On the bending moment capability on the pressurized abdominal cavity during human lifting activity. Ergonomics 1988,31, 817-828.

41. Tortora GJ. Principles of Human Anatomy. Eight edition. New York: John Wiley \& Son, 1999. 